\title{
Mitochondrial assembly: protein import
}

\author{
David A. Hood* and Anna-Maria Joseph \\ School of Kinesiology and Health Science and Department of Biology, York University, Toronto, \\ Ontario M3J 1P3, Canada
}

\begin{abstract}
The protein import process of mitochondria is vital for the assembly of the hundreds of nuclearderived proteins into an expanding organelle reticulum. Most of our knowledge of this complex multisubunit network comes from studies of yeast and fungal systems, with little information known about the protein import process in mammalian cells, particularly skeletal muscle. However, growing evidence indicates that the protein import machinery can respond to changes in the energy status of the cell. In particular, contractile activity, a powerful inducer of mitochondrial biogenesis, has been shown to alter the stoichiometry of the protein import apparatus via changes in several protein import machinery components. These adaptations include the induction of cytosolic molecular chaperones that transport precursors to the matrix, the up-regulation of outer membrane import receptors, and the increase in matrix chaperonins that facilitate the import and proper folding of the protein for subsequent compartmentation in the matrix or inner membrane. The physiological importance of these changes is an increased capacity for import into the organelle at any given precursor concentration. Defects in the protein import machinery components have been associated with mitochondrial disorders. Thus, contractile activity may serve as a possible mechanism for up-regulation of mitochondrial protein import and compensation for mitochondrial phenotype alterations observed in diseased muscle.
\end{abstract}

Skeletal muscle: Exercise: Protein import: Mitochondrial disease

The assembly of an organelle as intricate as the mitochondrion requires the integration of several vital processes, including the transcription of hundreds of mitochondrial-associated genes, the translocation and targetting of newly synthesized proteins and the assembly of proteins into a functional unit. Since the mitochondrial genome is limited in the number of genes it can encode, the nucleus, in coordination with the mitochondria, is given the crucial task of being the major provider of these essential polypeptides. The important function of trafficking these nuclear-transcribed and cytosolically-synthesized proteins into the organelle is carried out by several translocation processes, each consisting of a distinct network of multisubunit proteins, collectively referred to as the mitochondrial protein import machinery (Truscott et al. 2003). Why is the study of mitochondrial assembly and protein import so important? The failure to incorporate an appropriate combination of gene products can lead to reduced synthesis of ATP, or the accelerated production of reactive oxygen species, both of which can lead to cell death, or mitochondrial disease.

\section{Mitochondria exist as different subfractions within striated muscle}

The mitochondrion is subdivided into four compartments, including the intermembrane space, the matrix and the outer and inner membranes. In striated muscle mitochondria exist within separate cellular compartments as two functionally and biochemically distinct groups: subsarcolemmal (SS) mitochondria, which accumulate beneath the sarcolemma; intermyofibrillar (IMF) mitochondria, which are found interspersed among the myofibrils (Hoppeler, 1986; Cogswell et al. 1993). In general, numerous studies indicate that, while the SS mitochondria are more concentrated and closely opposed to each other around the perimeter of the cell, they occupy a lower percentage of the total mitochondrial volume (approximately 10-20,

\footnotetext{
Abbreviations: Hsp, heat-shock protein; IMF, intermyofibrillar; SS, subsarcolemmal; Tim, component proteins of the translocases of the inner mitochondrial membrane; Tom, component proteins of TOM; TOM, translocases of the outer mitochondrial membrane. * Corresponding author: Dr David A. Hood, fax +14167365698 , email dhood@yorku.ca
} 
depending on tissue and species) than the IMF mitochondria (Hoppeler, 1986). It is also well established that SS mitochondria are more adaptable than IMF mitochondria, in that the concentration of SS mitochondria is readily changed in response to conditions of muscle use and disuse (Hood, 2001). In addition, in certain mitochondrial diseases the histochemical appearance of ragged-red fibres is a result of the abnormal proliferation of SS mitochondria under the sarcolemma (Rifai et al. 1995). Thus, in response to the same cellular stimulus SS mitochondrial volume expansion within the cell is differentially affected compared with IMF mitochondria.

\section{Protein targetting to the mitochondria}

Initial studies of mitochondrial protein import were performed in the yeast Saccharomyces cerevisiae and the fungus Neurospora crassa. These investigations continue to contribute immensely to our understanding of the components of the protein import machinery. In mammalian cells work has progressed much more slowly, with research just beginning to identify the essential components of this complex protein trafficking system.

The process begins with the synthesis of precursor proteins, which are transcribed in the nucleus, escorted to the mitochondria and unfolded via a specialized group of molecular chaperones within the cytosol (Komiya et al. 1996; Fig. 1). The most important of these chaperones are the cytosolic heat-shock protein (Hsp) 70 and mitochondrial import stimulation factor. These chaperones interact with precursor proteins depending on the presence of specific targetting information within the primary structure of the protein. The targetting signal can be found as cleavable $\mathrm{N}$-terminal extensions termed presequences, or embedded within the mature polypeptide as a noncleavable sequence (Neupert, 1997). The type of signal contained in the precursor protein not only dictates its localization within the mitochondria, but also the translocation pathway that it will take and its energy requirement. For example, ATP-dependent mitochondrial import stimulation factor-mediated import shows a preference for preproteins with an internal signal, whereas cytosolic Hsp70 translocation is not ATP dependent and binds to preproteins containing a presequence (Komiya et al. 1996; Fig. 1).

\section{The translocases of the outer mitochondrial membrane machinery}

A multimeric receptor complex termed the translocases of the outer mitochondrial membrane (TOM; comprising component proteins (Tom); Fig. 1) exists in the outer mitochondrial membrane to interact with chaperones and their associated precursor proteins (also termed precursors). This complex is the only entry gate for precursors destined for the mitochondria. Precursors are recognized by the three receptor proteins Tom 20 , Tom 22, and Tom70 present on the outer mitochondrial surface. Tom70 generally binds to precursor proteins with an internal targetting signal (Neupert, 1997; Pfanner \& Geissler,
2001). A tetratricopeptide repeat domain present in Tom70 acts as a landing site for cytosolic chaperones, thereby facilitating the translocation of precursor proteins across the outer membrane (Young et al. 2003). These protein-protein interaction domains have also been identified in several other TOM components, including Tom20 (Abe et al. 2000) and Tom34 (Nuttall et al. 1997).

The remaining TOM complex is composed of Tom40 and the accessory proteins Tom5, Tom6 and Tom7 (Dekker et al. 1998). Together with Tom22, these proteins form a $400 \mathrm{kDa}$ conducting channel known as the general import pore (Dekker et al. 1998; van Wilpe et al. 1999). Using blue native gel electrophoresis, Model et al. (2001) have shown in yeast that the biogenesis of the $400 \mathrm{kDa}$ general import pore into the outer membrane is preceded by two assembly intermediates of 250 and $100 \mathrm{kDa}$. This technique should prove useful in future work to establish the steps in the assembly of multisubunit complexes in mammalian cells, and whether these steps can be accelerated under conditions of mitochondrial biogenesis.

Additional components implicated in the mammalian translocation pathway include OM37 and Tom34. OM37 is a $37 \mathrm{kDa}$ protein of the outer membrane that serves as a docking site for mitochondrial import stimulation factormediated precursor proteins (Komiya et al. 1996). In contrast, Tom 34 is characterized as a peripheral membrane protein primarily found within the cytosol (Chewawiwat et al. 1999). Although the precise role of this protein remains enigmatic, its association with numerous cytosolic proteins suggests that it may have a chaperone-like role to facilitate precursor import into mitochondria (Yang \& Weiner, 2002).

\section{The translocases of the inner mitochondrial membrane machinery}

The next portion of the translocation pathway involves the movement of precursors from the TOM complex across the intermembrane space to the inner membrane. This process involves a component protein (Tim) of the translocases of the inner mitochondrial membrane complex, Tim50; a protein with a single transmembrane segment consisting of a large hydrophilic domain located in the intermembrane space (Mokranjac et al. 2003). Tim50 appears to interact with translocation intermediates destined for the matrix, and it is involved in transferring precursors between the TOM and translocases of the inner mitochondrial membrane complexes (Geissler et al. 2002; Yamamoto et al. 2002). The Tim 23 complex consists of Tim 23 , Tim 17 and Tim44 subunits, and it serves to accept precursors containing a cleavable N-terminal sequence, and direct their entry into the inner membrane or the matrix (Pfanner \& Geissler, 2001; Fig. 1). This process is facilitated by several lower-molecular-weight proteins in the intermembrane space, such as Tim8 and Tim13, that serve as chaperones to pass precursors to the Tim23 complex (Leuenberger et al. 1999). In contrast, preproteins such as metabolite carriers with internal targetting signals do not enter the matrix via the Tim 23 channel, but transverse the intermembrane space to be directed to the Tim22 complex, and are subsequently incorporated into the inner 


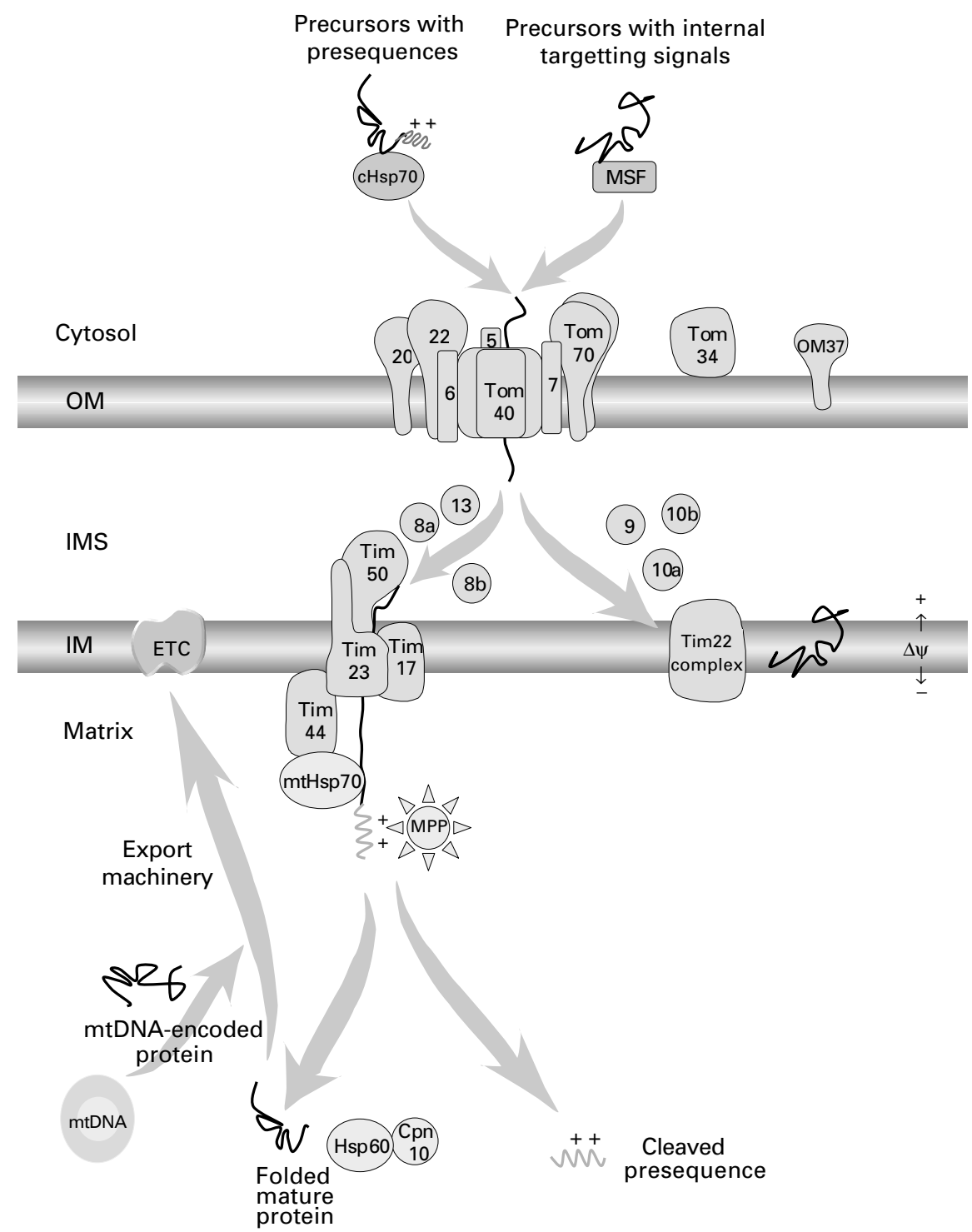

Fig. 1. Protein import into mitochondria. The translocases of the outer mitochondrial membrane (TOM) complex consists of component proteins (Tom) that include three receptor proteins, Tom20, Tom22 and Tom70, as well as Tom40, Tom5, Tom6 and Tom7. Precursors with a cleavable $\mathrm{N}$-terminal presequence are directed from the cytosol to the outer membrane $(\mathrm{OM})$ by cytosolic heat-shock protein (Hsp) 70. Precursors with internal targetting signals are chaperoned to the TOM complex by mitochondrial import stimulation factor (MSF). The precursor associates with Tom20, Tom22 and Tom70 receptors and is transferred to the intermembrane space (IMS) via the Tom40 complex. One of the component proteins (Tim) of the translocases of the inner membrane (IM) complex, Tim50, and the smaller Tim isoforms subsequently direct the precursor either to the Tim22 channel to be inserted into the IM, or to the Tim23 channel to be pulled into the matrix via the ATP-driven action of mitochondrial Hsp70 (mtHsp70) and the membrane potential $(\Delta \psi)$. Once inside the matrix, the presequence is cleaved by mitochondrial processing peptidase, and refolded by Hsp60 and its co-chaperonin chaperonin (Cpn) 10 into a mature protein. Subsequently, both nuclear and mitochondrially-encoded proteins are inserted into the IM by the mitochondrial export machinery. ETC, electron transport chain. (Modified from Hoogenraad et al. 2002; Truscott et al. 2003.)

membrane. Similar to Tim23, this complex consists of several other proteins, including Tim9 and Tim10, which transfer precursors to Tim22 (Koehler et al. 1998; Sirrenberg et al. 1998).

\section{Translocation, processing and assembly events in the matrix}

Once precursors enter the Tim 23 channel, they are pulled into the matrix by mitochondrial Hsp70 using the energy 
derived from the hydrolysis of ATP. Tim44 is a closely associated protein that binds mitochondrial Hsp70, thereby increasing the concentration of mitochondrial Hsp70 at the translocation site (Rassow et al. 1994; Schneider et al. 1994). The mechanism by which mitochondrial Hsp70 drives preproteins into the matrix is still under considerable debate. This translocation across the inner membrane is also dependent on the electrophoretic effect of the membrane potential (inside negative) on the positivelycharged presequence (Martin et al. 1991). In addition, the phospholipid cardiolipin in the inner mitochondrial membrane is also imperative for protein translocation, since it appears to orient the precursor in the correct position to interact with the Tim44-mitochondrial Hsp70 complex (Leenhouts et al. 1996).

Once the preprotein enters the matrix, the N-terminal signal sequence is cleaved by the mitochondrial processing peptidase (Gakh et al. 2002). Some precursors require additional processing, and this reaction is catalysed by a second metalloprotease, the mitochondrial intermediate peptidase (Isaya et al. 1992; Kalousek et al. 1992). The resulting mature protein possesses a lower molecular weight than the precursor protein, and is refolded by Hsp60 and its co-chaperonin chaperonin 10 (Fig. 1). The mature protein can then be assembled into functional components of the electron transport chain. This assembly step is done in combination with protein subunits derived from mitochondrial DNA, and is facilitated by the export machinery of the mitochondria (Fig. 1) that translocates proteins from the matrix to the inner membrane. Several protein export and assembly machinery components have been identified, including oxidase assembly 1 , cytochrome c oxidase 18 (Truscott et al. 2003) and SURF-1 (Tiranti et al. 1998). The absence of SURF-1 in man is the primary cause of Leigh syndrome, a severe neurodegenerative disorder (Tiranti $e t$ al. 1998). The effects of exercise on the expression of this class of assembly proteins have not yet been investigated.

\section{Mitochondrial protein import in muscle}

Studies with mitochondria isolated from skeletal muscle were initially designed to explore the possibility that the different biochemical features of SS and IMF mitochondria are, in fact, attributable to altered rates of protein import. Isolated IMF mitochondria have been found to exhibit rates of protein import that are 2-3-fold greater than those in SS mitochondria (Takahashi \& Hood, 1996). These rates closely match differences in mitochondrial respiration and ATP production. When mitochondrial respiration is experimentally reduced using known inhibitors of the electron transport chain, rates of import are correspondingly decreased. In addition, when interaction of the precursor protein with the phospholipid cardiolipin is impaired with adriamycin, protein import is reduced, particularly in the SS mitochondria. This effect corresponds with the higher cardiolipin content in SS, compared with IMF mitochondria (Takahashi \& Hood, 1996).

\section{Protein import and contractile activity}

Chronic exercise, or experimentally-induced contractile activity, are powerful stimuli for mitochondrial biogenesis in muscle. Part of the reason for this adaptation is now known to be the result of alterations in the expression of protein import machinery components (Ornatsky et al. 1995; Takahashi et al. 1998; Gordon et al. 2001). Among the changes shown to be induced are increases in cytosolic chaperones Hsp70 and mitochondrial import stimulation factor, as well as intramitochondrial chaperones Hsp60, mitochondrial Hsp70 and chaperonin 10 (Ornatsky et al. 1995; Neufer et al. 1996; Takahashi et al. 1998). In addition, cardiolipin, a unique phospholipid found within the inner mitochondrial membrane, is rapidly increased with chronic contractile activity. This change occurs at a rate that is faster than the increase in mitochondrial protein accumulation (Takahashi \& Hood, 1993), suggesting that the assembly of the mitochondrial reticulum in response to exercise occurs first by the synthesis of membrane phospholipids, followed subsequently by the insertion of membrane proteins. This pattern is similar to that found in other experimental systems (Hallman \& Kankare, 1971; Aithal \& Tustanoff, 1975).

In relation to outer membrane import receptors, Takahashi et al. (1998) have shown that Tom 20 protein levels are increased following $7 \mathrm{~d}$ of contractile activity. These changes are functionally relevant, since they correspond with an acceleration of malate dehydrogenase and mitochondrial transcription factor A import rates into the mitochondrial matrix (Takahashi et al. 1998; Gordon et al. 2001). The increased rate of import of mitochondrial transcription factor A into the matrix is particularly noteworthy, since this protein is an essential mitochondrial DNA transcription factor. Once imported, mitochondrial transcription factor A promotes the transcription and replication of the mitochondrial genome, leading to increased mitochondrial DNA copy number (Williams et al. 1986) and increased transcription of cytochrome c oxidase subunits (Gordon et al. 2001).

Thus, chronic contractile activity promotes mitochondrial biogenesis in part because of the induction of: (1) cytosolic molecular chaperones that transport precursors to the matrix; (2) outer membrane import receptors such as Tom20; (3) inner membrane phospholipids; (4) matrix chaperonins such as mitochondrial Hsp70, which pulls the precursors into the matrix in 'ratchet-like' fashion; (5) matrix chaperonins that refold the mature protein for subsequent compartmentation in the matrix or inner membrane. The physiological importance of these adaptations is an increased capacity for import (i.e. an increase in the maximum velocity of the transport process). As with any other transport or enzymic step, this increase in capacity represents an improved sensitivity at any given substrate (i.e. precursor protein) concentration (Fig. 2). In this case the precursor protein is provided within the cell by the combined efforts of both transcription and translation. Thus, even if these processes were unaffected by contractile activity, the increased capacity for import would result in a greater translocation of existing cytosolic precursor proteins into the mitochondrial matrix. An alternative interpretation is that the increased capacity for import would permit lower rates of transcription and translation, but would allow the same rate of import into the organelle (Fig. 2). 


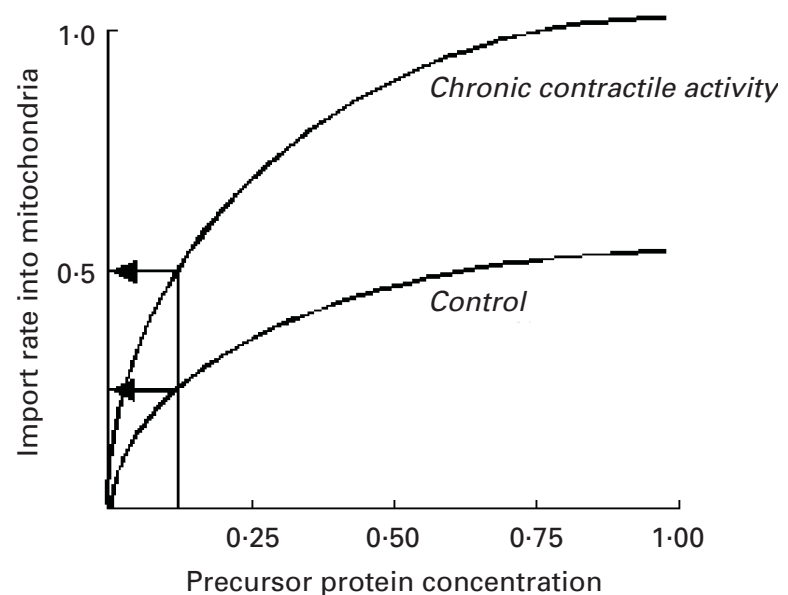

Fig. 2. Hypothetical response of contractile activity-induced changes in protein import. At any given concentration of precursor protein, contractile activity will result in a greater import rate into the mitochondrial matrix. Alternatively, the same rate of protein import can be achieved at a lower concentration of precursor protein provided by upstream transcriptional and translational events.

Interestingly, this adaptation does not hold true for all precursors. The import of $\mathrm{Bcl}-2$ into the mitochondrial outer membrane remains unchanged by identical contractile activity conditions that produce an increased import into the matrix (Takahashi et al. 1998). Further work on this compartment-specific adaptation is required in order to examine import rates into the intermembrane space and inner membrane.

It is also noteworthy that most of the effects of contractile activity on mitochondrial protein import have also been shown to occur in cardiac cells in response to thyroid hormone treatment (Craig et al. 1998; Schneider $\&$ Hood, 2000). This finding reinforces the concept that adaptations in the protein import pathway are a normal component of the adaptive responses of the organelle during mitochondrial biogenesis, even to markedly different stimuli.

\section{Importance of specific protein import machinery components}

The role of assigning a specific function to individual components of the protein import machinery has proven difficult in mammalian cells because of the lack of availability of cloned mammalian homologues. The first subunit to be identified was the surface receptor protein Tom20 (Goping et al. 1995; Seki et al. 1995). In yeast cells human Tom 20 has been shown to complement respiratory defects in cells lacking this protein (Goping et al. 1995; Seki et al. 1995). Overexpression of Tom20 by 2-3-fold in $\mathrm{C} 2 \mathrm{C} 12$ muscle cells grown in culture results in a parallel increase in the import of malate dehydrogenase, suggesting that Tom 20 alone could accelerate protein import into the matrix (Grey et al. 2000). In addition, a forced reduction in Tom 20 expression using antisense oligonucleotides leads to equivalent decrements in import, measured in intact cells using immunoprecipitation of the radiolabelled precursor protein (Grey et al. 2000). Antibody inhibition studies have also substantiated this finding, since pre-incubation of isolated mitochondria with a Tom 20 antibody has been shown to inhibit the import of matrix proteins malate dehydrogenase and mitochondrial transcription factor $\mathrm{A}$, but to have little effect on the import of Bcl-2 (Grey et al. 2000). Thus, the import of matrix-destined proteins appears to be largely dependent on the expression of Tom20. Whether other outer membrane protein components share similar importance is not yet established in mammalian cells. However, the critical role of Tom 20 for import adds relevance to the study of Tom20 expression. The upstream promoter region of the human Tom 20 gene contains potential binding sites for nuclear respiratory factors 1 and 2 (Hernandez et al. 1999), which are known to be vital for mitochondrial biogenesis (Scarpulla, 2002). Since these transcription factors are transactivated by PPAR $\gamma$ co-activator- $1 \alpha$, and this protein is rapidly increased by contractile activity (Irrcher et al. 2003), this process may be a mechanism for the exerciseinduced increase in Tom 20 expression, and protein import, that occurs.

Similar overexpression experiments with mitochondrial Hsp70 in H9C2 myocytes have been performed to examine the potential role of this protein in the import pathway. Surprisingly, 2-3-fold increases in mtHsp70 levels using stably transfected cells produce only a very modest $13 \%$ increase in import (Colavecchia et al. 2003). This finding suggests that mitochondrial Hsp70 is normally found in excess within the organelle, and additional levels of the protein contribute little to the enhancement of the import rate. Further work to establish the threshold level of mitochondrial Hsp70, as well as other import machinery components using RNA interference analysis is needed to identify the critical proteins involved in the adaptation of muscle cells to stimuli that evoke mitochondrial biogenesis.

\section{The importance of protein import in mitochondrial disorders}

The aetiology of mitochondrial disorders can be classified into two categories: those arising from genetic mutations in the nuclear genome; those attributed to mutations in mitochondrial DNA (Zeviani et al. 1998). Given the importance of protein import into mitochondria, it is quite possible that mutations arising in the nuclear genes encoding the protein import machinery can reduce the energy supply within a cell and lead to mitochondrial disease. In the fungus Podospora anserina a mutation in the Tom70 receptor has been shown to result in mitochondrial DNA deletions during senescence (Jamet-Vierny et al. 1997). In man a defect in the gene encoding the deafness and dystonia protein 1 has been shown to result in a neurodegenerative disorder termed Mohr-Tranebjaerg syndrome (Koehler et al. 1999). Deafness and dystonia protein 1 is homologous to the yeast Tim8p family of proteins that is responsible for escorting proteins from the intermembrane space into the matrix (Koehler et al. 1999). Deafness and dystonia protein 1 (Tim8a) interacts with Tim13 to aid in 
the translocation of Tim23 (Rothbauer et al. 2001). The mutation in deafness and dystonia protein 1 caused by a cysteine $\rightarrow$ tryptophan transition (C66W) impairs its proper folding, leading to the inability of deafness and dystonia protein 1 to associate with Tim13, and the insufficient import of Tim23 into the inner membrane (Hofmann et al. 2002).

Patients with multiple mitochondrial defects exhibit an altered expression of several Hsp. Analyses of skeletal muscle tissue from patients with mitochondrial myopathies reveal distinct differences in the expression of Hsp60 among the two mitochondrial subfractions of skeletal muscle (Carrier et al. 2000). Hsp60 levels are reduced in SS mitochondria, while IMF mitochondria display an overabundance of the protein. Interestingly, Hsp60 is undetectable in ragged-red fibres with a proliferation of SS mitochondria, suggesting that the proteins may be vital for normal organelle biogenesis (Carrier et al. 2000). In another study fibroblasts from a patient with multiple mitochondrial disease were examined to determine whether protein import defects could account for the impaired mitochondrial function found in this condition (Rungi et al. 2001). In contrast to what was originally believed, protein import rates into mitochondria were found to be normal. These normal rates coincided with unaltered levels of Tom20, but markedly reduced levels of both mitochondrial Hsp70 and Hsp60. Once again, these data point to an important role for Tom20 in maintaining the rate of protein import, even in conditions of mitochondrial dysfunction.

Cultured cells partially, or completely, depleted of mitochondrial DNA ( $\rho^{-}$or $\rho^{0}$ respectively) by ethidium bromide treatment have also been used as a model of mitochondrial disease (Desjardins et al. 1985; King \& Attardi, 1989). These cells, which exhibit impaired membrane potential and reduced ATP synthesis, display an up-regulation of many nuclear-encoded genes, including components of the protein import machinery (Martinus et al. 1996). Interestingly, protein import does not appear to be impaired (Nelson \& Schatz, 1979; Herzberg et al. 1993) in these respiratory-deficient cells, suggesting the involvement of a compensatory response of the protein import apparatus to maintain protein import and mitochondrial assembly under conditions of energetic deficiency. More work relating the energy state of the cell and protein import in specific human mitochondrial diseases is warranted.

\section{Summary}

Mitochondrial biogenesis requires the cooperative interaction of both the nuclear and the mitochondrial genomes. The mitochondrial protein import pathway is responsible for translocating the hundreds of nuclear-derived proteins, and it is a vital step in the expansion of the mitochondrial reticulum. Protein import is a process that is inducible in response to exercise (Takahashi et al. 1998; Gordon et al. 2001). This adaptation is brought about by the altered expression of several of the protein import machinery components such as Tom20, which appears to be one of the most important proteins within this translocation pathway. The identification of more mammalian homologues, along with their functional characterization in both healthy muscle and muscle from patients with mitochondrial disease, will help in the evaluation of the potential role of exercise in improving any import pathway defects that may exist.

\section{Acknowledgements}

The research in the authors' laboratory is supported by the Canadian Institutes of Health Research, and the Natural Science and Engineering Research Council of Canada. D.A.H. holds a Canada Research Chair in Cell Physiology.

\section{References}

Abe Y, Shodai T, Muto T, Mihara K, Torii H, Nishikawa S, Endo $\mathrm{T}$ \& Kohda D (2000) Structural basis of presequence recognition by the mitochondrial import receptor Tom20. Cell 100, 551-560.

Aithal HN \& Tustanoff ER (1975) Assembly of complex III into newly developing mitochondrial membranes. Canadian Journal of Biochemistry 53, 1278-1281.

Carrier H, Flocard F, Tagliati V, Arrigo AP \& Godinot C (2000) Immunolabelling of mitochondrial superoxide dismutase and of Hsp60 in muscles harbouring a respiratory chain deficiency. Neuromuscular Disorders 10, 144-149.

Chewawiwat N, Yano M, Terada K, Hoogenraad NJ \& Mori M (1999) Characterization of the novel mitochondrial protein import component, Tom34, in mammalian cells. Journal of Biochemistry (Tokyo) 125, 721-727.

Cogswell AM, Stevens RJ \& Hood DA (1993) Properties of skeletal muscle mitochondria isolated from subsarcolemmal and intermyofibrillar regions. American Journal of Physiology 264, C383-C389.

Colavecchia M, Christie LN, Kanwar YS \& Hood DA (2003) Functional consequences of thyroid hormone-induced changes in the mitochondrial protein import pathway. American Journal of Physiology 284, E29-E35.

Craig EE, Chesley A \& Hood DA (1998) Thyroid hormone modifies mitochondrial phenotype by increasing protein import without altering degradation. American Journal of Physiology 275, C1508-C1515.

Dekker PJ, Ryan MT, Brix J, Muller H, Honlinger A \& Pfanner N (1998) Preprotein translocase of the outer mitochondrial membrane: molecular dissection and assembly of the general import pore complex. Molecular and Cellular Biology 18, $6515-6524$

Desjardins P, Frost E \& Morais R (1985) Ethidium bromideinduced loss of mitochondrial DNA from primary chicken embryo fibroblasts. Molecular and Cellular Biology 5, $1163-1169$

Gakh O, Cavadini P \& Isaya G (2002) Mitochondrial processing peptidases. Biochimica et Biophysica Acta 1592, 63-77.

Geissler A, Chacinska A, Truscott KN, Wiedemann N, Brandner K, Sickmann A, Meyer HE, Meisinger C, Pfanner N \& Rehling $P$ (2002) The mitochondrial presequence translocase: an essential role of Tim50 in directing preproteins to the import channel. Cell 111, 507-518.

Goping IS, Millar DG \& Shore GC (1995) Identification of the human mitochondrial protein import receptor, huMas20p. Complementation of delta mas20 in yeast. FEBS Letters $\mathbf{3 7 3}$, $45-50$. 
Gordon JW, Rungi AA, Inagaki H \& Hood DA (2001) Effects of contractile activity on mitochondrial transcription factor A expression in skeletal muscle. Journal of Applied Physiology 90, 389-396.

Grey JY, Connor MK, Gordon JW, Yano M, Mori M \& Hood DA (2000) Tom20-mediated mitochondrial protein import in muscle cells during differentiation. American Journal of Physiology 279, C1393-C1400.

Hallman M \& Kankare P (1971) Cardiolipin and cytochrome $\alpha \alpha_{3}$ in intact liver mitochondria of rats. Evidence of successive formation of inner membrane components. Biochemical and Biophysical Research Communications 45, 1004-1010.

Hernandez JM, Giner P \& Hernandez-Yago J (1999) Gene structure of the human mitochondrial outer membrane receptor Tom20 and evolutionary study of its family of processed pseudogenes. Gene 239, 283-291.

Herzberg NH, Middelkoop E, Adorf M, Dekker HL, Van Galen MJ, Van den Berg M, Bolhuis PA \& Van den Bogert C (1993) Mitochondria in cultured human muscle cells depleted of mitochondrial DNA. European Journal of Cell Biology 61, 400-408.

Hofmann S, Rothbauer U, Muhlenbein N, Neupert W, Gerbitz KD, Brunner M \& Bauer MF (2002) The C66W mutation in the deafness dystonia peptide 1 (DDP1) affects the formation of functional DDP1.TIM13 complexes in the mitochondrial intermembrane space. Journal of Biological Chemistry 277, 23287-23293.

Hood DA (2001) Contractile activity-induced mitochondrial biogenesis in skeletal muscle. Journal of Applied Physiology 90, 1137-1157.

Hoogenraad NJ, Ward LA \& Ryan MT (2002) Import and assembly of proteins into mitochondria of mammalian cells. Biochemica et Biophysica Acta 1592, 97-105.

Hoppeler H (1986) Exercise-induced ultrastructural changes in skeletal muscle. International Journal of Sports Medicine 7, 187-204.

Irrcher I, Adhihetty PJ, Sheehan T, Joseph AM \& Hood DA (2003) PPARgamma coactivator-1alpha expression during thyroid hormone- and contractile activity-induced mitochondrial adaptations. America Journal of Physiology 284, C1669-C1677.

Isaya G, Kalousek F \& Rosenberg LE (1992) Amino-terminal octapeptides function as recognition signals for the mitochondrial intermediate peptidase. Journal of Biological Chemistry 267, 7904-7910.

Jamet-Vierny C, Contamine V, Boulay J, Zickler D \& Picard M (1997) Mutations in genes encoding the mitochondrial outer membrane proteins Tom70 and Mdm10 of Podospora anserina modify the spectrum of mitochondrial DNA rearrangements associated with cellular death. Molecular and Cellular Biology 17, 6359-6366.

Kalousek F, Isaya G \& Rosenberg LE (1992) Rat liver mitochondrial intermediate peptidase (MIP): purification and initial characterization. EMBO Journal 11, 2803-2809.

King MP \& Attardi G (1989) Human cells lacking mtDNA: repopulation with exogenous mitochondria by complementation. Science 246, 500-503.

Koehler CM, Jarosch E, Tokatlidis K, Schmid K, Schweyen RJ \& Schatz G (1998) Import of mitochondrial carriers mediated by essential proteins of the intermembrane space. Science 279, 369-373.

Koehler CM, Leuenberger D, Merchant S, Renold A, Junne T \& Schatz G (1999) Human deafness dystonia syndrome is a mitochondrial disease. Proceedings of the National Academy of Sciences USA 96, 2141-2146.

Komiya T, Sakaguchi M \& Mihara K (1996) Cytoplasmic chaperones determine the targeting pathway of precursor proteins to mitochondria. EMBO Journal 15, 399-407.
Leenhouts JM, Torok Z, Mandieau V, Goormaghtigh E \& de Kruijff B (1996) The N-terminal half of a mitochondrial presequence peptide inserts into cardiolipin-containing membranes. Consequences for the action of a transmembrane potential. FEBS Letters 388, 34-38.

Leuenberger D, Bally NA, Schatz G \& Koehler CM (1999) Different import pathways through the mitochondrial intermembrane space for inner membrane proteins. EMBO Journal 18, 4816-4822.

Martin J, Mahlke K \& Pfanner N (1991) Role of an energized inner membrane in mitochondrial protein import. Delta psi drives the movement of presequences. Journal of Biological Chemistry 266, 18051-18057.

Martinus RD, Garth GP, Webster TL, Cartwright P, Naylor DJ, Hoj PB \& Hoogenraad NJ (1996) Selective induction of mitochondrial chaperones in response to loss of the mitochondrial genome. European Journal of Biochemistry 240, 98-103.

Model K, Meisinger C, Prinz T, Wiedemann N, Truscott KN, Pfanner N \& Ryan MT (2001) Multistep assembly of the protein import channel of the mitochondrial outer membrane. Nature Structural Biology 8, 361-370.

Mokranjac D, Paschen SA, Kozany C, Prokisch H, Hoppins SC, Nargang FE, Neupert W \& Hell K (2003) Tim50, a novel component of the TIM23 preprotein translocase of mitochondria. EMBO Journal 22, 816-825.

Nelson N \& Schatz G (1979) Energy-dependent processing of cytoplasmically made precursors to mitochondrial proteins. Proceedings of the National Academy of Sciences USA $\mathbf{7 6}$, 4365-4369.

Neufer PD, Ordway GA, Hand GA, Shelton JM, Richardson JA, Benjamin IJ \& Williams RS (1996) Continuous contractile activity induces fiber type specific expression of HSP70 in skeletal muscle. American Journal of Physiology 271, C1828-C1837.

Neupert W (1997) Protein import into mitochondria. Annual Review of Biochemistry 66, 863-917.

Nuttall SD, Hanson BJ, Mori M \& Hoogenraad NJ (1997) hTom34: a novel translocase for the import of proteins into human mitochondria. DNA and Cell Biology 16, 1067-1074.

Ornatsky OI, Connor MK \& Hood DA (1995) Expression of stress proteins and mitochondrial chaperonins in chronically stimulated skeletal muscle. Biochemical Journal 311, 119-123.

Pfanner N \& Geissler A (2001) Versatility of the mitochondrial protein import machinery. Nature Reviews Molecular Cell Biology 2, 339-349.

Rassow J, Maarse AC, Krainer E, Kubrich M, Muller H, Meijer M, Craig EA \& Pfanner N (1994) Mitochondrial protein import: biochemical and genetic evidence for interaction of matrix hsp70 and the inner membrane protein MIM44. Journal of Cell Biology 127, 1547-1556.

Rifai Z, Welle S, Kamp C \& Thornton CA (1995) Ragged red fibers in normal aging and inflammatory myopathy. Annals of Neurology 37, 24-29.

Rothbauer U, Hofmann S, Muhlenbein N, Paschen SA, Gerbitz KD, Neupert W, Brunner M \& Bauer MF (2001) Role of the deafness dystonia peptide 1 (DDP1) in import of human Tim23 into the inner membrane of mitochondria. Journal of Biological Chemistry 276, 37327-37334.

Rungi AA, Primeau A, Nunes Christie L, Gordon JW, Robinson BH \& Hood DA (2001) Events upstream of mitochondrial protein import limit the oxidative capacity of fibroblasts in multiple mitochondrial disease. Biochimica et Biophysica Acta 1586, $146-154$.

Scarpulla RC (2002) Transcriptional activators and coactivators in the nuclear control of mitochondrial function in mammalian cells. Gene 286, 81-89. 
Schneider HC, Berthold J, Bauer MF, Dietmeier K, Guiard B, Brunner M \& Neupert W (1994) Mitochondrial Hsp70/MIM44 complex facilitates protein import. Nature 371, 768-774.

Schneider JJ \& Hood DA (2000) Effect of thyroid hormone on mtHsp70 expression, mitochondrial import and processing in cardiac muscle. Journal of Endocrinology 165, 9-17.

Seki N, Moczko M, Nagase T, Zufall N, Ehmann B, Dietmeier K, Schafer E, Nomura N \& Pfanner N (1995) A human homolog of the mitochondrial protein import receptor Mom19 can assemble with the yeast mitochondrial receptor complex. FEBS Letters 375, 307-310.

Sirrenberg C, Endres M, Folsch H, Stuart RA, Neupert W \& Brunner M (1998) Carrier protein import into mitochondria mediated by the intermembrane proteins Tim10/Mrs11 and Tim12/Mrs5. Nature 391, 912-915.

Takahashi M, Chesley A, Freyssenet D \& Hood DA (1998) Contractile activity-induced adaptations in the mitochondrial protein import system. American Journal of Physiology 274, C1380-C1387.

Takahashi M \& Hood DA (1993) Chronic stimulation-induced changes in mitochondria and performance in rat skeletal muscle. Journal of Applied Physiology 74, 934-941.

Takahashi M \& Hood DA (1996) Protein import into subsarcolemmal and intermyofibrillar skeletal muscle mitochondria. Differential import regulation in distinct subcellular regions. Journal of Biological Chemistry 271, 27285-27291.

Tiranti V, Hoertnagel K, Carrozzo R, Galimberti C, Munaro M, Granatiero M et al. (1998) Mutations of SURF-1 in Leigh disease associated with cytochrome c oxidase deficiency. American Journal of Human Genetics 63, 1609-1621.

Truscott KN, Brandner K \& Pfanner N (2003) Mechanisms of protein import into mitochondria. Current Biology 13, R326-R337.

van Wilpe S, Ryan MT, Hill K, Maarse AC, Meisinger C, Brix J, Dekker PJ, Moczko M, Wagner R, Meijer M, Guiard B, Honlinger A \& Pfanner N (1999) Tom22 is a multifunctional organizer of the mitochondrial preprotein translocase. Nature 401, 485-489.

Williams RS, Salmons S, Newsholme EA, Kaufman RE \& Mellor J (1986) Regulation of nuclear and mitochondrial gene expression by contractile activity in skeletal muscle. Journal of Biological Chemistry 261, 376-380.

Yamamoto H, Esaki M, Kanamori T, Tamura Y, Nishikawa S \& Endo T (2002) Tim50 is a subunit of the TIM23 complex that links protein translocation across the outer and inner mitochondrial membranes. Cell 111, 519-528.

Yang CS \& Weiner H (2002) Yeast two-hybrid screening identifies binding partners of human Tom34 that have ATPase activity and form a complex with Tom34 in the cytosol. Archives of Biochemistry and Biophysics 400 , $105-110$.

Young JC, Hoogenraad NJ \& Hartl FU (2003) Molecular chaperones Hsp90 and Hsp70 deliver preproteins to the mitochondrial import receptor Tom70. Cell 112, 41-50.

Zeviani M, Tiranti V \& Piantadosi C (1998) Mitochondrial disorders. Medicine 77, 59-72. 